\title{
Perceptions locales du changement climatique et mesures d'adaptation dans la gestion des parcs à karité au Nord-Bénin
}

\author{
Paul Césaire GNANGLE ${ }^{1 *}$, Janvier EGAH ${ }^{2}$, Mohamed Nasser BACO ${ }^{2}$, \\ Charlemagne D. S. J. GBEMAVO ${ }^{3}$, Romain Glèlè KAKAÏ ${ }^{3}$ et Nestor SOKPON ${ }^{2}$ \\ ${ }^{1}$ Agronome socio-économiste, Agrométéorologiste du développement, agroforestier, Chercheur au Programme \\ de Recherche Forestière (PRF) du Centre de Recherche Agricole du Centre (CRAC) de l'Institut National des \\ Recherches Agricoles du Bénin (INRAB), 01 P 884 Cotonou, Bénin, Tél : +229 95282199 \\ ${ }^{2}$ Faculté d'Agronomie, Université de Parakou, BP 123, Parakou, Bénin. \\ ${ }^{3}$ Faculté des Sciences Agronomiques, Université d'Abomey - Calavi, O1BP 526 Tri Postal, Cotonou, Bénin. \\ *Auteur correspondant, E-mail : gnampaces@yahoo.fr
}

\section{RESUME}

Les perceptions paysannes du changement climatique, les stratégies d'adaptation dans la gestion des parcs à karité sont étudiées au Nord-Bénin. Au total, 120 unités de recherche sont enquêtées dans 6 villages choisis en fonction de la densité de pieds de karité dans le terroir du village, de l'importance représentée par le karité pour les groupes socioculturels. Les données collectées concernaient les perceptions et les déterminants socioéconomiques. Les données sont analysées avec le test d'indépendance de $\chi^{2}$ et l'Analyse Factorielle des Correspondances. Les résultats ont permis d'identifier 17 perceptions liées aux changements climatiques et 16 stratégies d'adaptation. Les stratégies d'adaptation dépendaient des niveaux de prospérité mais n'avaient pas de relation avec l'appartenance aux clases d'âges. Les paysans percevaient le changement climatique dans les parcs à karité à travers la poche de sécheresse, le brouillard, la chute des rendements des cultures en association avec le karité, le bouleversement de la phénologie du karité et la prolifération des guis sur le karité. Les stratégies d'adaptation développées face au changement climatique par les producteurs sont relatives aux pratiques magico-religieuses, l'enlèvement de gui sur le karité, l'adaptation de nouvelles cultures en association, la protection des jeunes plants de karité et l'élagage.

(C) 2012 International Formulae Group. All rights reserved.

Mots clés : Perceptions, stratégies d'adaptation, parcs à karités, changement climatique, Bénin.

\section{INTRODUCTION}

Le monde entier est caractérisé par le phénomène de variabilité climatique ces 30 dernières années. Cette variabilité climatique se manifeste essentiellement par la hausse de la température et la diminution de la hauteur des précipitations. Guibert et al. (2010) ont montré que l'analyse conventionnelle des 
séries de pluviométrie, de température et de vitesse du vent confirme l'augmentation des températures maximales et minimales mais ne permet pas la mise en évidence de différence dans les répartitions des pluies ni de l'augmentation des vents violents au Nord Bénin. Les données issues des stations météorologiques entre 1960 et 2008 montrent que la hauteur moyenne de pluie a connu une régression de $3,2 \mathrm{~mm} / \mathrm{an}$ et la température a connu une tendance linéaire d'élévation avec un accroissement de $0,03{ }^{\circ} \mathrm{C} / \mathrm{an}$ (Gnanglè et al., 2011). Ces manifestations ne sont pas restées sans conséquences sur l'agriculture pluviale des pays de l'Afrique de l'Ouest comme le Bénin, tributaire des saisons. L'agriculture de ces pays contribue pour près de $30 \%$ au PIB, emploie plus de $50 \%$ de la population active (Renard et al., 2004) et est fortement dépendante du climat.

Les paysages agrosylvicoles sont des sources de revenus pour des populations locales et entrent dans la conservation de l'environnement. Au Bénin, dans la liste des paysages agrosylvicoles rencontrés figurent les parcs à karité et néré. Dans ces parcs les arbres de karité et de néré sont disposés dans un arrangement aléatoire. Le karité revêt une importance socioculturel, économique, médicinal, alimentaire et agroforestier (Codjia et al., 2003; Gnanglè, 2005; Sodjinou, 2006; Diarassouba et al., 2008; Gnanglè et al., 2009; Gnanglè, 2010). Les estimations du MAEP (2008) montrent que le karité constitue le troisième produit d'exportation après le coton et l'anacarde au Bénin, et que la part annuelle apportée par le karité au revenu national est de 1,05 milliards de francs CFA. Le fruit du karité est consommé par les populations tant rurales qu'urbaines. La pulpe du fruit contient de grandes quantités de protéines, de minéraux et les amandes sont riches en acide gras comme les acides oléique, stéarique, linoléique et palmitique (Wiesman et Maranz, 2001). Le beurre obtenu à partir des noix de karité est consommé localement ou exporté pour les cosmétiques, la confiserie, la fabrication du chocolat, la pâtisserie et les margarines végétales (Gnanglè, 2005). Diarassouba et al. (2008) ont montré que des tradipraticiens emploient les produits du karité pour traiter en moyenne 43 affections. En outre, l'arbre améliore le microclimat et la fertilité du sol pour les récoltes des cultures qui lui ont associées; les branches sont utilisées comme bois de chauffage (Bayala et al., 2002). En Afrique, certains travaux sont effectués de façon globale sur les perceptions paysannes du changement climatique notamment ceux de Brou et al. (2005) en Côte d'Ivoire et ceux de Aho et al. (2008) et de Gnanglè et al. (2009) au Centre du Bénin. Les perceptions et stratégies d'adaptation du changement climatique sur les systèmes agroforestiers à base de karité dans la zone de prédilection du karité ne sont quasiment pas étudiées. Ce qui soulève les questions suivantes: Comment les acteurs du monde agricole, les paysans perçoivent-ils ces changements climatiques et comment réagissent-ils? $\mathrm{Au}$ regard des grandes importances des parcs à karité, il paraît important d'analyser l'impact du changement climatique sur les parcs à karité du Bénin afin de mieux les conserver.

\section{MATERIEL ET METHODES}

L'étude a été réalisée dans le NordBénin, une zone de transition soudanoguinéenne et une zone soudanienne, dans trois des cinq parcs à karité et néré identifiés par Gbédji (2003) et Gnanglè (2005). Ces parcs ont été choisis en fonction de leur niveau de production en fruits et de la densité des arbres. Il s'agit des parcs de Parakou, de Bembèrèké et de Kandi. Au sein de chaque parc, une 
commune a été choisie à savoir : commune de Bassila dans le parc de Parakou ; commune de Bembèrèké dans le parc de Bembèrèké; commune de Banikoara dans le parc de Kandi. L'ensemble de la zone d'étude s'étend de $9^{\circ} 45^{\prime}$ à $12^{\circ} 25^{\prime}$ latitude Nord (Figure 1). Cette zone est caractérisée par une pluviométrie annuelle dont la moyenne oscille entre 1.000 et $1.200 \mathrm{~mm}$. L'humidité relative moyenne maximale varie entre 76 et $85 \%$ tandis que l'insolation moyenne journalière oscille entre 7 et 8,4 heures et dixième. Ces caractéristiques climatiques déterminent un gradient climatique Nord-Sud. Les types de sols rencontrés dans le milieu d'étude sont les sols minéraux peu évolués, sols ferrugineux peu lessivés, sols hydromorphes, sols ferralitiques faiblement dénaturés servant de support à toutes les cultures pluviales, sols ferrugineux tropicaux lessivés et sols ferrugineux tropicaux appauvris (Gbédji, 2003).

Le milieu d'étude est le domaine des mosaïques de forêts claires, éventuellement de forêts denses sèches, parsemées de savanes arborées et arbustives avec la présence des parcs agroforestiers à karité et néré, de roneraies, etc. Il comprend des pseudosteppes, des galeries forestières avec des arbres de plus petite taille recouvrant faiblement le sol. Dans les formations naturelles et anthropiques, les espèces couramment rencontrées sont Afzelia africana, Anogeissus leiocarpa, Daniellia oliveri, Ceiba pentandra, Isoberlinia doka, Parkia biglobosa, Pterocarpus erinaceus, Vitellaria paradoxa et Borassus aethiopim.

Les trois communes retenues ont été choisies après plusieurs entretiens avec des personnes ressources telles que des agents forestiers, agents du Programme de Conservation et de Gestion des Ressources Naturelles, responsables des sociétés d'achat d'amandes de karité et ses fournisseurs, responsables des projets de développement et Organisations Non Gouvernementales, etc.. Les propositions faites par les personnes ressources, reposent sur l'importance des pieds de karité dans la commune, la diversité de la population et l'importance que la population accorde au karité. Dans chaque commune, deux villages ont été choisis en utilisant les critères de Diarassouba et al. (2008). Ces critères sont relatifs à l'intensification des activités de transformation et de commercialisation du karité, à l'importance que les producteurs lui accordent dans le village, à la disponibilité des parcs à karité en exploitation, à la diversité socioéconomique et aux particularités socioculturelles (groupes socioculturels). Les villages choisis sont ceux de Manigri Ikani et de Frignon dans la commune de Bassila, de Wanrarou et de Sombouan dans la commune de Bembèrèké, de Sirikou et de l'Alibori B dans la commune de Banikoara.

Une méthodologie plurielle associant des techniques quantitatives et qualitatives a été utilisée pour sélectionner les unités d'observations. Les enquêtés ont été choisis de façon aléatoire selon leur disponibilité et de façon à couvrir toute la diversité socioéconomique des exploitations agricoles des villages d'étude. Au total, 120 enquêtés ont été choisis à raison de 30 par village. Par ailleurs, les fiches d'enquêtes ont été prétestées lors de cette phase exploratoire afin de les adapter aux réalités du terrain.

Les données collectées sont relatives aux caractéristiques socioéconomiques, aux perceptions des paysans sur le changement climatique et aux stratégies d'adaptation développées par les producteurs pour faire face aux changements climatiques dans la gestion des parcs à karité. Ces données ont été obtenues à partir des focus group et des 
entretiens semi-structurés et informels donnant une large manœuvre aux enquêtés. De même, des entretiens occasionnels ont été organisés pour solliciter des réponses d'éclaircissement dès l'apparition d'une nouvelle information suscitant des réflexions (Diarassouba et al., 2008).

A l'issue des enquêtes dans chaque village, les ménages étudiés ont été soumis à une classification par niveau de prospérité et par classe d'âge. Les classifications ont été faites par des informateurs clés choisis dans chaque village sur la base de leur âge et de leur connaissance des ménages du village. Les niveaux de prospérité retenus sont les pauvres, les riches et la classe intermédiaire entre pauvres et riches, la classe des moyens. Les très jeunes, les jeunes, les adultes et les personnes âgées sont les quatre classes d'âge retenues. Le sens des terminologies utilisées est ce qui suit :

1. Pauvres: les producteurs qui n'arrivent pas à satisfaire leurs besoins.

2. Moyens : les producteurs qui arrivent à satisfaire partiellement leurs besoins.

3. Riches: les producteurs qui arrivent à satisfaire leurs besoins.

4. Très jeunes : les producteurs ayant un âge compris entre 20 et 25 ans.

5. Jeunes: les producteurs ayant un âge compris entre 25 et 40 ans.

6. Adultes: les producteurs ayant un âge compris entre 40 et 60 ans.

7. Personnes âgées : les producteurs ayant un âge supérieur à 60 ans.

L'analyse de discours a été utilisée pour retranscrire les réponses des producteurs afin de faire ressortir les verbatim utilisés pour appréhender les perceptions et les stratégies d'adaptation au changement climatique. Le test d'indépendance de Chi-Square a servi à vérifier la dépendance ou non d'une part entre les perceptions et les caractéristiques socioéconomiques (niveaux de prospérité et classes d'âge) et d'autre part, entre les stratégies d'adaptation au changement climatique et les caractéristiques socioéconomiques. L'analyse factorielle des correspondances (AFC) a été utilisée pour relier les groupes de perceptions aux caractéristiques socioéconomiques.

\section{RESULTATS}

\section{Perceptions paysannes du changement climatique \\ Le changement climatique n'est pas} seulement constaté par les scientifiques puisque depuis plus de trois décennies, les populations locales du Nord-Bénin l'avait aussi perçu aisément. Au total, 17 perceptions du changement climatique ont été identifiées dans le Nord-Bénin (Tableau 1). Ces perceptions dépendaient des niveaux de prospérité $\left(\chi^{2}=69,12 ; \mathrm{P}=0,000 ; \mathrm{ddl}=32\right)$ mais pas des clases d'âges $\left(\chi^{2}=38,12 ; \mathrm{P}=\right.$ 0,248; ddl = 48).

Les perceptions les plus citées par les producteurs étaient la poche de sécheresse, l'harmattan et l'excès de chaleur. Ces perceptions relevaient des facteurs naturels $(88 \%)$ et facteurs socio-culturels (12\%). Quatre perceptions du changement climatique (la poche de sécheresse, l'harmattan, l'excès de chaleur et le tarissement des marigots) représentaient plus de la moitié des fréquences cumulées des réponses données par les producteurs sur leur perception du changement climatique. Les producteurs les appréciaient et les percevaient comme suit :

\section{La poche de sécheresse}

Les producteurs soulignaient qu'il y a une trentaine d'année, au démarrage des pluies en avril, il était rare d'observer 2 à 3 jours sans pluie. Toutefois, aujourd'hui, après le démarrage tardif des pluies vers fin mai, des arrêts de pluie dans les mois de juin et d'août 
sont non seulement constatés mais peuvent atteindre une vingtaine de jours.

\section{L'harmattan}

Le déplacement de la période d'apparition de l'harmattan, le raccourcissement des délais et la variation de l'intensité de l'harmattan d'une année à l'autre sont des signes du changement climatique. «Le chauffage au feu de bois, est une pratique qui disparaît de nos jours. L'harmattan se féminise de nos jours, elle n'est plus forte ». Ces propos d'un paysan de Bembèrèkè confirmaient la lecture du changement climatique que les populations faisaient à travers l'harmattan.

\section{Excès de chaleur}

Les producteurs expriment l'excès de chaleur par le raccourcissement de la durée de la chaleur et l'augmentation de son intensité.

\section{Tarissement des marigots}

Les producteurs montrent clairement que l'eau dans les marigots débordait pendant la période de pluies (juin-août). Ainsi, l'eau restait jusqu'en saison sèche pour abreuver les animaux. Ce qui n'est plus le cas de nos jours. D'où l'expression du tarissement des marigots selon eux.

Typologie des perceptions paysannes en fonction des niveaux de prospérité

La projection des niveaux de prospérité et des perceptions paysannes dans le système d'axes issus de l'Analyse Factorielle des Correspondances (AFC) est faite sur la Figure

2. Dans le système d'axes 1 et 2 , toutes $(100 \%)$ les informations contenues dans les variables sont contrôlées (Figure 2). Les perceptions les plus liées au premier axe étaient la chute de rendement des cultures, les vents violents et la rareté des pluies, tandis que les perceptions telles que le retard des pluies, la poche de sécheresse, le brouillard, le prolongement des pluies, la phénologie du karité et le non respect des normes sociales étaient celles prises en compte par le deuxième axe. Le niveau de prospérité et les paysans moyennement prospères ont plus contribué à la formation du premier axe, tandis que les niveaux de prospérité pauvre et riche sont plus liés à l'axe 2. En reliant les perceptions paysannes et les niveaux de prospérité ayant le plus contribué à la formation des deux axes, les riches s'opposaient aux pauvres et exprimaient différemment les perceptions du changement climatique. En effet, les riches percevaient les changements climatiques par le retard de pluie, le brouillard et les poches de sécheresse tandis que les pauvres les percevaient à travers le non respect des normes sociales et le bouleversement de la phénologie du karité (Figure 2). Les paysans ayant un niveau de prospérité moyen percevaient les changements climatiques par les vents violents et la rareté des pluies. Les autres perceptions ne discriminaient pas les niveaux de prospérité.

\section{Typologie des perceptions paysannes en fonction des classes d'âge}

Les résultats de l'Analyse Factorielle des Correspondances (AFC) ont mis en relation les classes d'âge et les perceptions paysannes du changement climatique (Figure 3). Les informations contenues dans les variables sont contrôlées à $88 \%$ par le système d'axes 1 et 2 (Figure 3). Les perceptions du changement climatique telles que l'excès de chaleur, les vents violents, le brouillard et la rareté des pluies étaient celles ayant le plus contribué à la formation du premier axe tandis que l'harmattan, le retard des pluies et la chute de rendement sont les perceptions les plus liées au second axe. Concernant les classes d'âge, les paysans très jeunes et personnes âgées ont plus contribué à la formation du premier axe, tandis que les classes d'âge 
adultes et vieux sont plus liées à l'axe 2. En reliant les perceptions paysannes et les classes d'âge ayant le plus contribué à la formation des deux axes, les personnes âgées s'opposaient aux très jeunes en exprimant différemment les perceptions du changement climatique. En effet, les vieux percevaient les changements climatiques par le brouillard et le vent violent tandis que les très jeunes les percevaient à travers la chaleur et la rareté des pluies (Figure 3). Les paysans adultes percevaient les changements climatiques par les retards des pluies et la chute de rendement des cultures. Les autres perceptions ne discriminaient pas les classes d'âge parce qu'elles ne sont pas significativement exprimées sur les axes.

\section{Stratégies d'adaptation au changement climatique dans la gestion des parcs à karité}

Face aux conséquences néfastes du changement climatique sur les parcs à karité, les producteurs ont développé plusieurs stratégies pour s'adapter et réduire les risques climatiques (Tableau 2). A ces stratégies endogènes, s'ajoutaient celles relatives au paquet technologique vulgarisé par les institutions de recherche et de développement (fumure minérale, herbicide et semences améliorées). Le changement de culture, l'augmentation du nombre de sarclage, la fumure minérale et le ressemis étaient les quatre stratégies dont trois endogènes occupant plus de la moitié de l'ensemble des stratégies développées dans la zone d'étude (Tableau 2).

Les stratégies d'adaptation au changement climatique sont significativement liées aux niveaux de prospérité des producteurs $\left(\chi^{2}=61,45 ; \mathrm{P}=0,000 ; \mathrm{ddl}=30\right)$ tandis qu'elles n'étaient pas significativement dépendantes des classes d'âge $\left(\chi^{2}=36,53 ; \mathrm{P}=\right.$ 0,308; $\mathrm{ddl}=45)$. Selon les tendances observées le plus souvent, les stratégies d'adaptation variaient selon les groupes ethniques, l'âge, le sexe et les activités socioéconomiques des ménages. Toutefois, l'âge et le sexe n'étaient pas forcément les facteurs déterminant les stratégies adaptatives développées au niveau local mais plutôt l'expérience des paysans en matière d'agriculture et les capacités des ménages à avoir accès au crédit et au marché, donc à leur niveau de prospérité. De même, le développement de nouvelles idées pour agir sur la nature a entraîné des sociétés fondées non plus sur des inégalités liées à l'âge, au sexe ou à la parenté comme dans les sociétés primitives, mais des inégalités associées à l'existence de castes ou de classes.

\section{Relation entre les stratégies d'adaptation et les perceptions du changement climatique dans la gestion des parcs à karité \\ Certaines stratégies d'adaptation} étaient en relation avec les perceptions du changement climatique. Concernant les stratégies d'adaptation dans la gestion des parcs à karité en réponse aux perceptions paysannes en dehors du brouillard, les paysans ont développé des techniques simples comme stratégies d'adaptation aux perceptions locales du changement climatique dans la gestion des parcs à karité du Nord-Bénin. Par exemple, face à la poche de sécheresse, les producteurs développaient des pratiques magicoreligieuses consistant surtout à la provocation de la pluie (Tableau 3). 
Tabbleau 1 : Perceptions paysannes du changement climatique.

\begin{tabular}{lcc}
\hline $\begin{array}{l}\text { Perceptions paysannes du changement } \\
\text { climatique }\end{array}$ & $\begin{array}{c}\text { Fréquences relatives des } \\
\text { réponses } \mathbf{( \% )}\end{array}$ & $\begin{array}{c}\text { Types de } \\
\text { facteurs }\end{array}$ \\
\hline Poche de sécheresse & 16,35 & Naturel \\
Harmattan & 14,54 & Naturel \\
Excès de chaleur & 12,86 & Naturel \\
Tarissement des marigots & 9,25 & Naturel \\
Brouillard & 7,93 & Naturel \\
Retard des pluies & 6,61 & Naturel \\
Prolongement des pluies & 5,29 & Naturel \\
Chute de rendement (Karité \& cultures) & 4,45 & Naturel \\
Rareté des pluies & 4,45 & Naturel \\
Phénologie du karité & 4,33 & Naturel \\
Vent violent & 3,85 & Naturel \\
Arrêt précoce des pluies & 3,25 & Naturel \\
Non respect des normes sociales & 2,04 & Naturel \\
Prolifération des guis & 1,68 & Naturel \\
Déforestation & 1,32 & Naturel \\
Période de carême (Musulman) & 0,96 & Socio-culturel \\
Période des fêtes (Tabaski et Gani) & 0,84 & Socio-culturel \\
Fréquence totale & 100 & - \\
\hline
\end{tabular}

Tableau 2. Stratégies d'adaptation dans la gestion des parcs à karité face au changement Climatique.

\begin{tabular}{lcl}
\hline Stratégies d'adaptation & $\begin{array}{c}\text { Fréquences relatives des réponses } \\
(\boldsymbol{\%})\end{array}$ & Origine \\
\hline Changement de culture & 24,62 & Endogène \\
Augmentation de nombre de sarclage & 9,89 & Endogène \\
Fumure minérale & 9,59 & Exogène \\
Ressemis & 9,08 & Endogène \\
Déplacement des dates de semis & 8,58 & Endogène \\
Pratiques magico-religieuses & 6,56 & Endogène \\
Valorisation des bas-fonds & 6,16 & Endogène \\
Elagage & 4,94 & Endogène \\
Herbicidage & 4,84 & Exogène \\
Protection des jeunes plants de karité & 4,44 & Endogène \\
Substitution de karité par l'anacardier & 4,04 & Endogène \\
Utilisation de semences améliorées & 3,33 & Exogène \\
Enlèvement de gui & 2,02 & Endogène \\
Relabour & 1,01 & Endogène \\
Migration & 0,50 & Endogène \\
Diversification des activités & 0,40 & Endogène \\
\hline Fréquence totale & 100 & - \\
\hline
\end{tabular}


Tableau 3. Stratégies d'adaptation en fonction des perceptions du changement climatique dans la gestion des parcs à karité

\begin{tabular}{|c|c|}
\hline Perceptions paysannes & Stratégies d'adaptation en réponse aux perceptions \\
\hline Poche de sécheresse & $\begin{array}{l}\text {-Pratiques magico-religieuses : consistent à la provocation des pluies } \\
\text { - Déplacement des dates de semis des cultures associées au karité } \\
\text { - Ressemis des cultures associées aux arbres de karité }\end{array}$ \\
\hline Brouillard & Pas de stratégies endogènes \\
\hline Chute de rendement & Fumure minérale et organique \\
\hline Phénologie du karité & $\begin{array}{l}\text { Elagage : consiste à réduire les vieilles branches et racines des arbres de } \\
\text { karité pour une nouvelle forme de vie }\end{array}$ \\
\hline Prolifération des guis & Enlèvement de gui \\
\hline
\end{tabular}




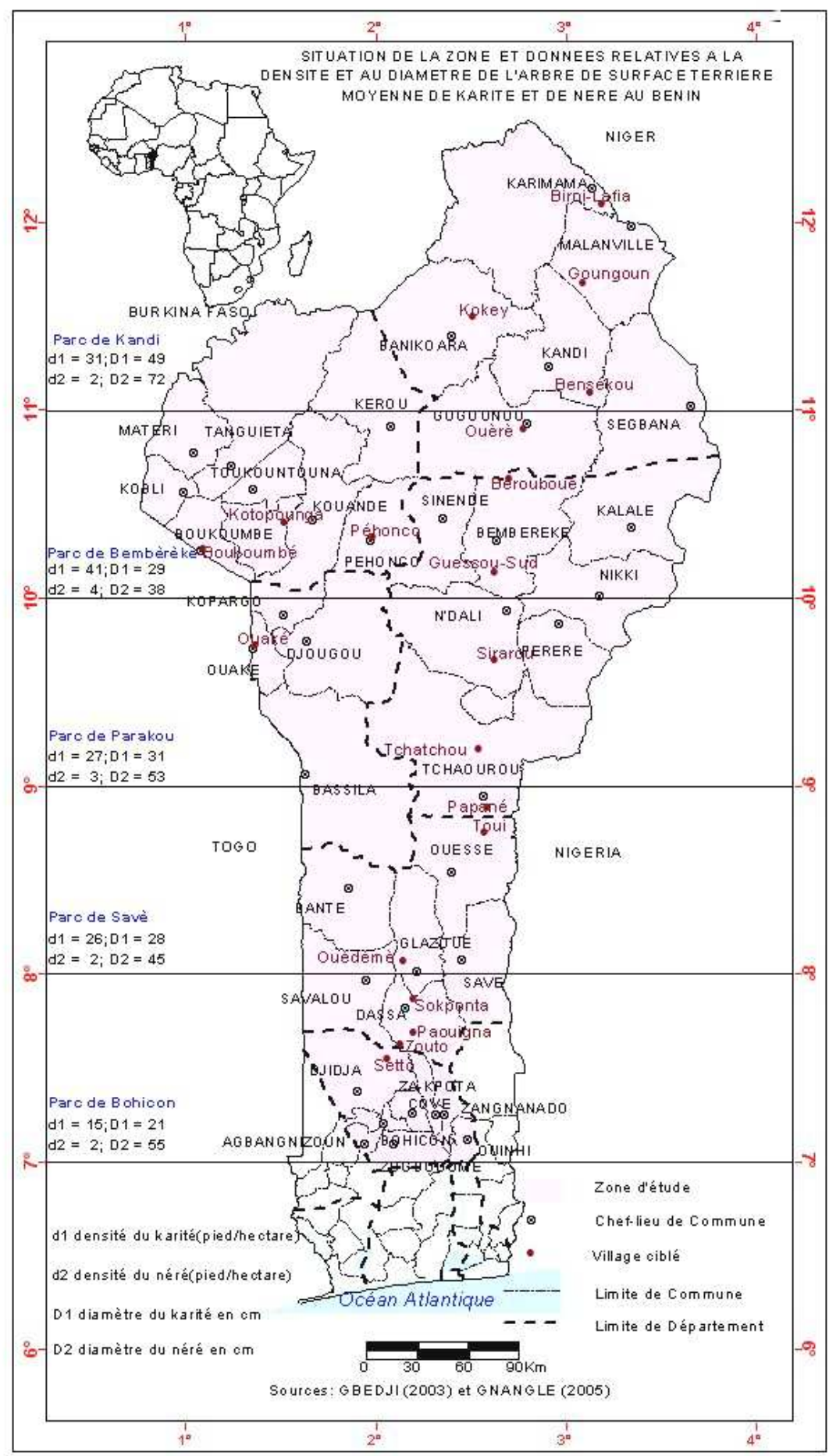

Figure 1 : Carte des parcs à karité et à néré au Bénin. 


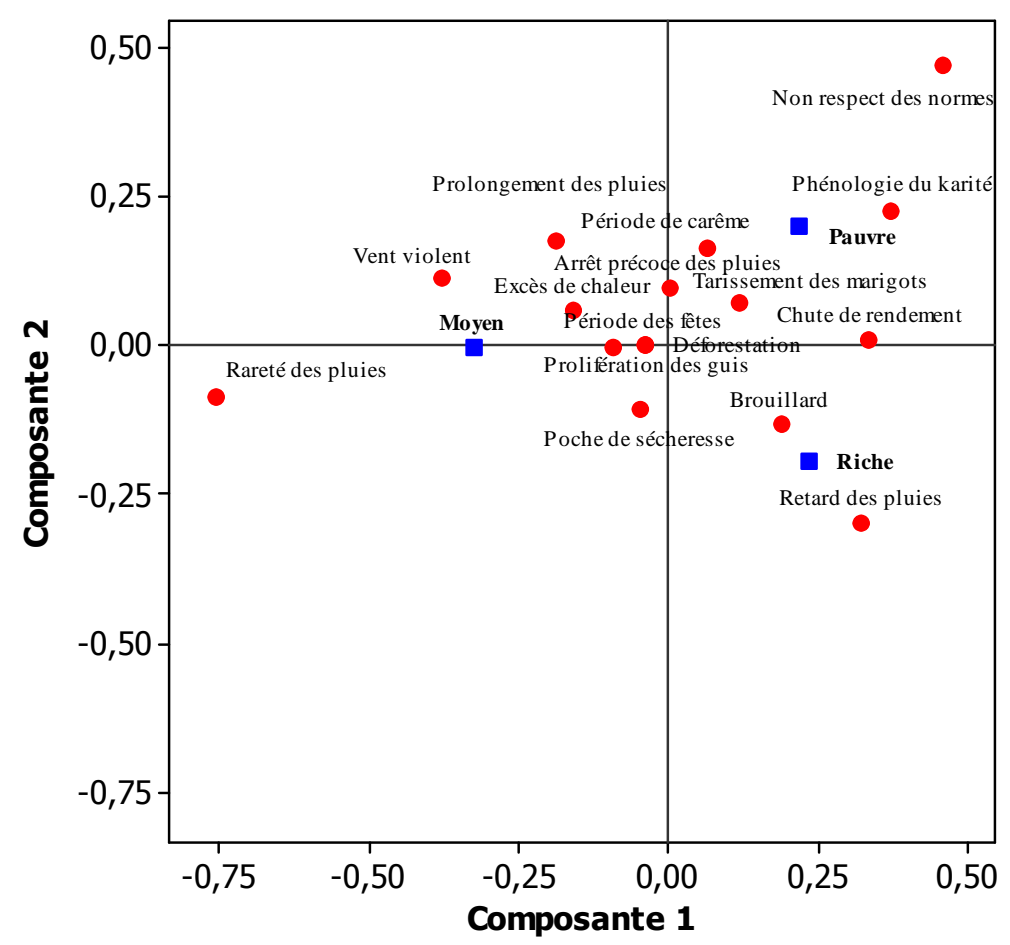

Figure 2 : Positionnement des perceptions paysannes et des niveaux de prospérité dans un système d'axes (Analyse Factorielle des Correspondances).

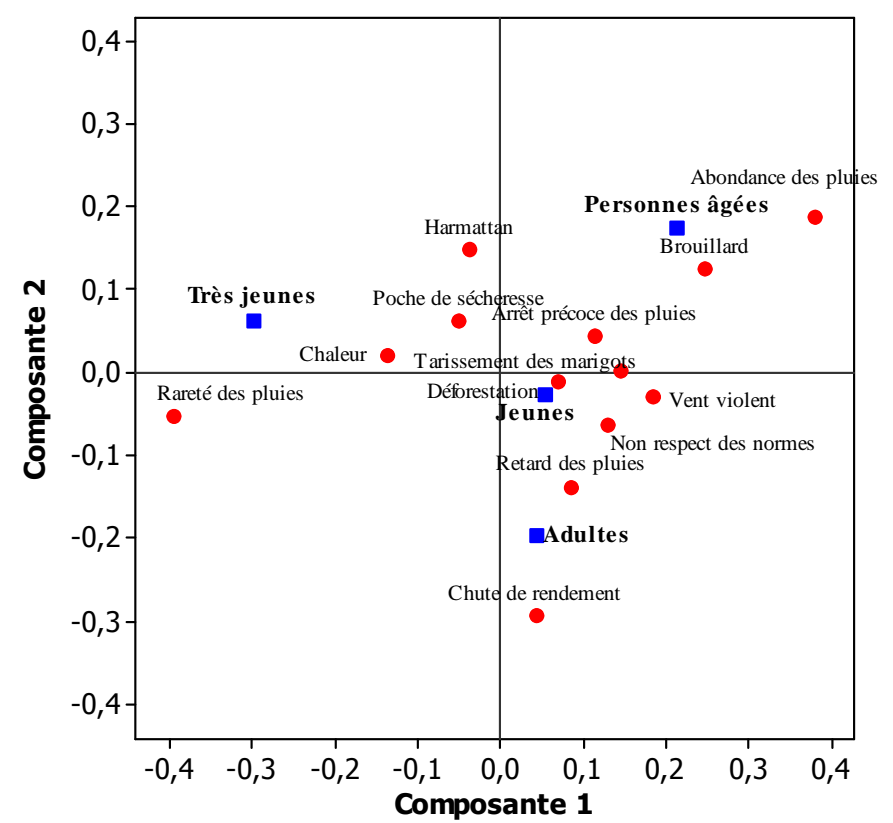

Figure 3 : Positionnement des perceptions paysannes et les classes d'âges dans un système d'axes (Analyse Factorielle des Correspondances). 


\section{DISCUSSION}

\section{Perceptions paysannes du changement climatique}

Le changement climatique à travers l'étude met en exergue plusieurs perceptions des producteurs. Les perceptions telles que les poches de sécheresse, les vents violents, la chaleur excessive, les pluies tardives révélées dans l'étude corroborent avec celles trouvées par PANA-Bénin (2008) dans les zones agroécologiques du Bénin et par Gnanglè et al. (2009) au Centre du Bénin. Les études de Hassan et Nhemechena (2008) et de Traoré et al. (2002) dans la sous-région ont montré que près de la moitié des producteurs ont perçu le changement climatique à travers l'élévation des températures à long terme et à la diminution des précipitations, à des changements prononcés de la synchronisation des pluies, à la récurrence des sécheresses et au tarissement des cours d'eau autrefois pérennes pendant la saison sèche. Ces résultats s'inscrivent dans le même ordre d'idée que ceux des producteurs de notre zone étude. En Côte d'Ivoire, les études de Brou et al. (2005) ont révélé le non respect des règles divines comme perception du changement climatique à travers la pratique de relations sexuelles discrètes en brousse, la profanation des lieux sacrés. Cette perception paysanne au Bénin s'exprime par des pratiques incestueuses.

Les perceptions paysannes trouvées dans l'étude et les résultats des scientifiques sur l'évolution des facteurs climatiques sont en adéquation en termes de variation de la température et de la pluviométrie. Les mêmes observations sont faites par les producteurs à travers la chaleur excessive, la rareté des pluies et les poches de sécheresse.

De cette étude, il ressort que les perceptions paysannes du changement climatique varient en fonction des niveaux de prospérité (pauvres, moyens et riches) et des classes d'âge (très jeunes, jeunes, adultes et personnes âgées). Nos résultats corroborent avec ceux de Teka et Vogt (2010), qui ont trouvé que les perceptions locales sur les risques naturels des habitants des zones côtières du Bénin varient suivant les groupes spécifiques (groupe social et âge). Concernant les niveaux de prospérité, ce sont les pauvres et les moins prospères qui expriment mieux les perceptions du changement climatique surtout sur les parcs à karité. Cela s'explique par le fait que ces catégories de producteurs sont celles qui ont comme principale activité l'agriculture. S'agissant des classes d'âge, les très jeunes et les jeunes n'ont pas une bonne perception du changement climatique du fait de leur âge. Selon Sánchez-Cortés et Lazos Chavero (2011) les adultes et particulièrement les personnes âgées, font recours au calendrier d'ethno-climatologie afin de comparer des changements détectés dans la variabilité du climat par contre, les jeunes ne mentionnent pas clairement ce calendrier ou leur connaissance ethno-météorologique en prévoyant le temps. Les jeunes ont peu d'expérience dans l'exploitation des parcs à karité pour mieux discerner les variations climatiques sur ces parcs. Sánchez-Cortés et Lazos Chavero (2011) ont confirmé à travers leur étude que l'expérience fait partie de la logique de la signification pour expliquer les changements environnementaux courants, $\mathrm{y}$ compris le climat.

\section{Stratégies d'adaptation, relations perceptions-stratégies face au changement climatique}

L'adaptation est un processus de gestion des risques climatiques en mettant en œuvre des mesures individuelles et collectives de prévention, de riposte et de relèvement (IAVS, 2011). Ce constat est fait dans l'étude dans la mesure où certaines stratégies d'adaptation sont appliquées collectivement. C'est le cas des pratiques magico-religieuses contrairement aux autres stratégies appliquées individuellement. La même remarque a été faite par Traoré et al. (2002) qui ont identifié 
des stratégies aussi bien collectives qu'individuelles. Selon ces auteurs, dans une région de Guinée, les producteurs ont développé des méthodes les plus appropriées de gestion de l'humidité du sol durant les périodes critiques du développement des plantes. Ainsi, des méthodes d'amélioration de la fertilité des sols, le choix des variétés plus résistantes à la sécheresse et le développement des cultures irriguées sont entre autres les stratégies utilisées par les producteurs de Guinée pour faire face au changement climatique. Ces stratégies s'assimilent à l'utilisation des semences améliorées, à la valorisation des bas-fonds et à l'application de la fumure minérale mises en œuvre par les producteurs de la zone d'étude pour faire face au changement climatique. Ceci vient confirmer l'assertion de Belliveau et al. (2006) selon laquelle le comportement adaptatif est relatif au lieu et au temps spécifique car en lieu et place du développement des cultures irriguées, les producteurs de la zone d'étude valorisent les bas-fonds au profit de l'igname. Ils se donnent plus à la culture de manioc compte tenu de la chute énorme de rendement de l'igname dans le contexte du changement climatique. Dans certaines communautés ivoiriennes, la rationalisation de la gestion de l'espace, de l'utilisation des intrants et du temps de travail (déplacement des dates de semis), la quête de la sécurité alimentaire par un ajustement des besoins aux disponibilités alimentaires et une diversification des sources de revenus sont des mesures de réponses que les producteurs ont développé pour faire face au changement climatique (Brou et al., 2005). Ce qui est observé dans le cadre de notre étude puisque les producteurs de la zone d'étude déplacent les dates de semis en fonction des pluies, utilisent de la fumure minérale et diversifient leurs sources de revenu. La quête de la sécurité alimentaire a été traduite ici par l'abandon peu à peu de l'igname au profit de manioc par les producteurs de la zone d'étude.
Ces communautés ivoiriennes organisent également de rituels d'imploration du pardon de Dieu pour faire revenir la pluie (Brou et al., 2005). Ceci se traduit ici par des pratiques magico-religieuses à travers lesquelles les producteurs provoquent la pluie. Cette stratégie a été élucidée à travers cette étude. Les mêmes résultats ont été trouvés par Teka et Vogt (2010), dans la gestion des risques naturels en zones côtière du Bénin où les populations locales ont développé des stratégies fondées sur des pratiques rituelles et culturelles manquant de rationalité scientifique et qui ne peuvent pas être ignorées par une gestion moderne et séculaire de la côte. L'adaptation au changement climatique ressort de la traduction des perceptions du changement climatique en des décisions agricoles (Bryant et al., 2000). Cette assertion est vérifiée dans notre étude puisque les producteurs de la zone d'étude mettent au point des stratégies d'adaptation à partir de leurs perceptions sur le changement climatique. Néanmoins, aucune stratégie n'est fondée pour faire face à la perception (brouillard). Ce qui peut s'expliquer par le fait que cette perception n'a pas d'effet direct sur les activités agricoles comme les autres perceptions.

\section{Conclusion}

Le changement climatique n'est pas seulement constaté par les scientifiques, mais perçu aussi par les producteurs. En effet, l'analyse des perceptions paysannes et des stratégies d'adaptation face au changement climatique dans la gestion des parcs à karité faite dans l'étude révèle que le changement climatique se perçoit non seulement à partir des facteurs naturels, mais aussi à travers les faits sociaux tels que les périodes des fêtes et des carêmes musulmans. Ces perceptions varient suivant les niveaux de prospérité et les classes d'âge. Face à la plupart de ces perceptions, des stratégies d'adaptation sont développées dans la gestion des parcs à karité 
afin de réduire les effets du changement climatique. Enfin, les producteurs développent des pratiques magico-religieuses consistant surtout à la provocation de la pluie face à la poche de sécheresse. Par conséquent, ces stratégies méritent d'être testées au plan scientifique afin d'évaluer leur performance. Ainsi, une fois confirmées et révélées efficaces, elles peuvent contribuer à la réduction de la vulnérabilité, à l'adaptation des sociétés et des écosystèmes au changement climatique.

\section{REMERCIEMENTS}

L'équipe de chercheurs adresse ces remerciements au Ministère français des Affaires Etrangères et Européenne à travers l'Agence Inter-Etablissements pour la Recherche et le Développement (AIRD), pour avoir financé cette recherche par le Fonds de Solidarité Prioritaire (FSP) dans le cadre du projet Recherche Interdisciplinaire et Participative sur les Interactions entre les Ecosystèmes, le Climat et les Sociétés d'Afrique de l'Ouest (RIPIECSA).

\section{REFERENCES}

Aho N, Boko M, Afouda A. 2008. Evaluation concertée de la vulnérabilité aux variations actuelles du climat et aux phénomènes météorologiques extrêmes. PANA/Bénin. 93p.

Bayala J, Teklehaimanot Z, and Ouedraogo SJ.2002. Millet production under pruned tree crowns in a parkland system in Burkina Faso. Agroforestry Systems., 54: 203-214.

Belliveau S, Bradshaw B, Smit B, Reid S, Ramsey D, Tarleton M, Sawyer B. 2006. Farm-level adaptation to multiple risks: Climate change and other concerns. Occasional paper No. 27. Canada: University of Guelph.

Brou YT, Akindès F, Bigot S. 2005. La variabilité climatique en Côte d'Ivoire : entre perceptions sociales et réponses agricoles. Cahiers Agricultures., 14(6): 533-540.

Bryant RC, Smit B, Brklacich M, Johnston RT, Smithers J, Chiotti Q, Singh B. 2000. Adaptation in Canadian agriculture to climatic variability and change. Climatic Change., 45: 181-201.

Codjia TJ, Assogbadjo EA, Ekué M RM. 2003. Diversité et valorisation au niveau local des ressources végétales forestières alimentaires du Bénin. Cahiers Agricultures., 12(5): 321-331.

Diarassouba N, Kouablan EK, Kanga AN, Patrick VD, Abdourahamane S. 2008. Connaissances locales et leur utilisation dans la gestion des parcs à karité en Côte d'Ivoire. Afrika focus., 21(1): 77-96.

Gbédji EKY. 2003. Caractérisation morphologique et structurale des parcs à néré (Parkia biglobosa (Jack;) R. Br. Ex. G. Dom.) au Bénin. Thèse d'Ingénieur Agronome, Université d'AbomeyCalavi, Bénin, p.124.

Gnanglè CP. Yabi AJ, Glèlè Kakaï JLR, Sokpon N. 2009. Changements climatiques : Perceptions et stratégies d'adaptations des paysans face à la gestion des parcs à karité au CentreBénin. www.sifee.org/Actes/actes_niamey .../1_GNANGLE_comm.pdf; 1-18.

Gnanglè PC, Glèlè Kakaï RL, Assogbadjo AE, Vodounon S, Yabi JA, Sokpon N. 2011. Tendances climatiques passées, modélisation, perceptions et adaptations locales au Bénin. Climatologie., 8: 26-40.

Gnanglè PC. 2005. Parcs à karité (Vitellaria paradoxa) (Gaertn. C. F.) (Sapotaceae) au Bénin: Importance socio-culturelle, caractérisations morphologique, structurale et régénération naturelle. Mémoire de DEA, Aménagement et Gestion des Ressources Naturelles, UAC/FSA, p.113.

Gnanglè PC. 2010. Management of shea butter in the context of climate change. International Innovation. Disseminating 
Science, Research and Technology. Environment, 54-55.

Guibert H, Allé UC, Dimon RO, Dédéhouanou H, Vissoh PV, Vodouhé SD, Tossou RC, Agbossou EK. 2010. Correspondance entre savoirs locaux et scientifiques: Perceptions des changements climatiques et adaptations au Bénin. ISDA 2010, Montpellier, 1-12.

Hassan R, Nhemachena C. 2008. Determinants of African farmers' strategies for adapting to climate change: multinomial choice analysis. AfJARE., 2(1): 83-104.

IAVS (Institut d'Application et de Vulgarisation en Sciences), 2011. Scénarios de processus paysans d'adaptation aux changements climatiques au Sahel et en Afrique de l'Ouest. Collection Science pour l'Agriculture, Ouagadougou, Burkina-Faso, 1-2.

MAEP. 2008. Atelier d'élaboration des stratégies des chaînes de valeur du karité au Bénin. Centre Guy Riobé de Parakou.

PANA-Bénin. 2008. Convention-Cadre Des Nations Unies Sur Les Changements Climatiques. Cotonou, Bénin, 1- 81.

Renard JF, Cheikh L, Knips V. 2004. L'élevage et l'intégration régionale en Afrique de l'Ouest. Ministère des Affaires étrangère, FAO-CIRAD, 1-37.
Sánchez-Cortés MS, · Lazos Chavero E. 2011. Indigenous perception of changes in climate variability and its relationship with agriculture in a Zoque community of Chiapas, Mexico. Climatic Change., 107: 363-389.

Sodjinou E. 2006. Institutions locales traditionnelles et modernes de gestion des ressources naturelles, des situations conflictuelles et divers conflits autour de ces ressources au Bénin. PAPA-INRABIFS, Bénin, 1-128.

Teka O et Vogt J. 2010. Social perception of natural risks by local residents in developing countries, The example of the coastal area of Benin. The Social Science Journal., 47: 215-224.

Traoré AF, Diallo ML, Bamba Z, Mara F. 2002. Communication initiale de la Guinée sur la Convention Cadre des Nations Unies sur les Changements Climatiques. Projet FEM/PNUD GUI/97/G33, Conakry, 1-87.

Wiesman Z, Maranz S. 2001. Chemical analysis of fruits of $\mathrm{V}$. paradoxa. In : Teklehaimanot, Z. (Ed). Improved management of agroforestry parklands systems in Sub-Saharian Africa EU/INCO Project Contract IC18-CT98-0261, Third Annual Progress Report, University of Wales Bangor, Uk, 81-92. 